\title{
Interaction-Induced Renormalization of Andreev Reflection
}

\author{
M. Titov, M. Müller, and W. Belzig \\ Department of Physics, University of Konstanz, D-78457 Konstanz, Germany
}

(Received 1 August 2006; published 8 December 2006)

\begin{abstract}
We analyze the charge transport between a one-dimensional weakly interacting electron gas and a superconductor within the scaling approach in the basis of scattering states. We derive the renormalization group equations, which fully account for the intrinsic energy dependence due to Andreev reflection. A strong renormalization of the corresponding reflection phase is predicted even for a perfectly transparent metal-superconductor interface. The interaction-induced suppression of the Andreev conductance is shown to be highly sensitive to the normal-state resistance, providing a possible explanation of experiments with carbon-nanotube/superconductor junctions by Morpurgo et al. [Science 286, 263 (1999)].
\end{abstract}

DOI: 10.1103/PhysRevLett.97.237006

The superconducting proximity effect has motivated a number of theoretical and experimental studies since the middle of the last century. Its low-energy physics is described by Andreev reflection processes [1] at the boundary between a normal metal $(\mathrm{N})$ and a superconductor $(\mathrm{S})$. In this process an electronlike quasiparticle in $N$ is reflected from the normal-metal-superconductor (NS) boundary as a hole, thus transferring a double electron charge $2 e$ into $\mathrm{S}$. The probability of such an event tends to unity in the case of an ideal NS interface provided the quasiparticle energy $\varepsilon$ is below the superconducting gap $\Delta$. Normal reflection takes place at nonideal interfaces due to the Fermi-energy mismatch in the superconducting and normal-metal materials or due to interface impurities.

In the elastic theory of electron transport, the NS boundary is characterized by energy-dependent quantummechanical amplitudes: $r^{A}(\varepsilon)$ for Andreev reflection and $r^{N}(\varepsilon)$ for normal reflection. The differential Andreev conductance of an NS junction measured at the voltage bias $V$ is given by [2]

$$
\frac{\partial I}{\partial V}=\frac{2 e^{2}}{h} \int d \varepsilon \frac{\partial f(\varepsilon-e V)}{\partial(e V)}\left(1+\left|r^{A}(\varepsilon)\right|^{2}-\left|r^{N}(\varepsilon)\right|^{2}\right),
$$

where $\varepsilon$ is measured with respect to the Fermi energy and $f(\varepsilon)$ is the Fermi distribution function for the temperature $T$. For $\varepsilon \gg \Delta$ the Andreev amplitude $r^{A}$ vanishes and Eq. (1) reduces to the Landauer formula for the conductance.

The Landauer formula as well as Eq. (1) play a key role in the scattering approach, which captures the effects of geometry, boundaries, and disorder in nanoscopic samples, but ignores inelastic quasiparticle scattering. In this Letter we apply Eq. (1) to a system consisting of a superconductor (for $x>0$ ) in contact with a one-dimensional electron gas (for $x<0$ ) with a repulsive interaction,

$$
\mathcal{H}_{I}=\frac{1}{2} \sum_{\sigma \sigma^{\prime}} \iint d x d y \Psi_{\sigma}^{\dagger}(x) \Psi_{\sigma^{\prime}}^{\dagger}(y) U_{x-y} \Psi_{\sigma^{\prime}}(y) \Psi_{\sigma}(x),
$$

PACS numbers: 74.45.+c, 71.10.Pm, 73.23.-b, 74.78.Na

where $U_{x}$ is a symmetric positive-definite function of $x$, which is assumed to exponentially decay for $x$ exceeding some characteristic interaction range $d$.

It is well known that electron-electron interactions in a one-dimensional Fermi gas strongly modify the spectrum of low-energy excitations [3]. In this case the elastic amplitudes $r^{N, A}(\varepsilon)$ in (1) have to be replaced by the renormalized ones $r_{D}^{N, A}(\varepsilon)$ with the cutoff $D=$ $\max \left\{\varepsilon, k_{B} T\right\}$. A way to calculate the renormalized scattering amplitudes was developed in Refs. [4,5], where the renormalization group (RG) equation for the $S$ matrix of a single barrier in a one-dimensional interacting electron gas was derived. The RG results are valid for arbitrary barrier transparencies but are restricted to the case of weak interactions. Complementary studies based on the bosonization technique [6-10] are applicable for arbitrary interaction strengths in the limits of very low or very high barrier transparency. The RG method of Refs. [4,5] was subsequently extended to account for a resonant energy dependence of the bare $S$ matrix [11,12]. We demonstrate below that the intrinsic energy dependence of Andreev reflection, which was disregarded in earlier works $[13,14]$, is crucial for understanding the transport properties of interacting normal-metal-superconductor nanostructures.

One experimental realization of a quasi-onedimensional electron system is provided by a single-wall carbon nanotube. In recent experiments [15] the differential Andreev conductance was dominated by the interface between a carbon nanotube and a superconductor. A strong deviation from the predictions of the elastic theory was observed at low temperatures and low voltage bias, in which case the Andreev conductance was found to be strongly suppressed. The effect, presumably due to electron-electron interactions, was shown to disappear with a slight change in the gate voltage applied to the nanotube. The major role of the gate voltage in the experimental setup is to change the nanotube-superconductor coupling and thereby the contact resistance. Thus, the experiment suggests a strong sensitivity of the interaction- 
induced dip in the Andreev conductance to the normalstate resistance of the NS interface.

Following the RG method of Refs. [11,12] we deal with an effective scattering matrix $S_{D}(\varepsilon)$, which acquires an additional dependence on the high-energy cutoff $D$. The renormalization procedure starts at a large $D=D_{0} \simeq$ $\hbar v_{F} / d$ with $v_{F}$ the Fermi velocity. The matrix $S_{D_{0}}$ coincides with the bare scattering matrix in the absence of interactions

$$
S_{D_{0}}(\varepsilon)=S(\varepsilon)=\left(\begin{array}{cc}
r(\varepsilon) & t^{\prime}(\varepsilon) \\
t(\varepsilon) & r^{\prime}(\varepsilon)
\end{array}\right) .
$$

In the $S$-matrix renormalization one starts with the analysis of the first order in $U$ correction to the $S$ matrix, which is regularized by a formal truncation of the Fourier series of Hartree and exchange potentials at the large momentum cutoff $D / \hbar v_{F}$. The first order correction is, then, proportional to $\ln (D / \varepsilon)$, showing the logarithmic divergence in the limit $\varepsilon \rightarrow 0$. By differentiating the first order result with respect to $D$ one arrives at the RG equation

$$
\frac{\partial S_{D}(\varepsilon)}{\partial \ln D}=\Sigma_{D}-S_{D}(\varepsilon) \Sigma_{D}^{\dagger} S_{D}(\varepsilon),
$$

which manifestly conserves the unitarity of the $S$ matrix [16]. The matrix $\Sigma_{D}$ depends on both $S_{D}(\varepsilon)$ and the renormalized interaction constants [17]

$$
g_{1}=\frac{\alpha_{1}}{1-2 \alpha_{1} \ln \left(D / D_{0}\right)}, \quad g_{2}=\alpha_{2}+\frac{g_{1}-\alpha_{1}}{2},
$$

which makes Eq. (4) a complex nonlinear equation. The bare values of the interaction constants

$$
\alpha_{1}=\int d x \frac{U_{x} e^{2 i k_{F} x}}{2 \pi \hbar v_{F}}, \quad \alpha_{2}=\int d x \frac{U_{x}}{2 \pi \hbar v_{F}},
$$

quantify backward and forward scattering, correspondingly. In order to guarantee the validity of Eq. (4) interactions are assumed to be weak, i.e. $\alpha_{1,2} \ll 1$. The RG procedure is terminated at $D=\max \left\{\varepsilon, k_{B} T\right\}$, so that the renormalized scattering matrix at zero temperature is given by $S_{\varepsilon}(\varepsilon)$.

For a single resonant tunnel barrier in an interacting quantum wire the RG equation for the $S$ matrix can be cast in the form of Eq. (4) with $[11,12]$

$$
\Sigma_{D}=\frac{1}{2}\left(\begin{array}{cc}
\left(2 g_{1}-g_{2}\right) r_{D}(-D) & 0 \\
0 & \left(2 g_{1}^{\prime}-g_{2}^{\prime}\right) r_{D}^{\prime}(-D)
\end{array}\right),
$$

where $g_{1,2}(D)$ and $g_{1,2}^{\prime}(D)$ refer to the interaction constants on the left and the right side of the barrier, correspondingly. The matrix $\Sigma_{D}$ takes into account the contribution to the Friedel oscillation from electrons with energy $-D$ deep in the Fermi sea. By lowering the cutoff according to Eq. (4) the coherent backward scattering from the electron density oscillation in the entire energy range is included in the RG procedure.

In the presence of superconductivity the dimension of the $S$ matrix has to be doubled. Therefore, the entries of
$S(\varepsilon)$ in the parametrization (3) have to be regarded as $2 \times 2$ matrices in Nambu space, for example,

$$
r(\varepsilon)=\left(\begin{array}{ll}
r^{N}(\varepsilon) & \bar{r}^{A}(\varepsilon) \\
r^{A}(\varepsilon) & \bar{r}^{N}(\varepsilon)
\end{array}\right)
$$

The components of $r(\varepsilon)$ fulfill an additional electron-hole symmetry constraint $\sigma_{y} r(-\varepsilon)^{*} \sigma_{y}=r(\varepsilon)$, where $\sigma_{y}$ is a Pauli matrix in Nambu space.

We find that the RG equation for the $S$ matrix of the NS interface takes the form (4) with

$$
\Sigma_{D}=\left(\begin{array}{cc}
\sigma_{D} & 0 \\
0 & 0
\end{array}\right)
$$

where $\sigma_{D}$ is the Nambu matrix

$$
\begin{aligned}
\sigma_{D} & =\frac{1}{2}\left(\begin{array}{cc}
\left(2 g_{1}-g_{2}\right) r_{D}^{N}(-D) & \left(g_{1}+g_{2}\right) \bar{s}(D) \\
\left(g_{1}+g_{2}\right) s(D) & \left(2 g_{1}-g_{2}\right) \bar{r}_{D}^{N}(D)
\end{array}\right), \\
s(D) & =\frac{1}{2}\left(r_{D}^{A}(-D)+r_{D}^{A}(D)\right) .
\end{aligned}
$$

The lower diagonal block of $\Sigma_{D}$ vanishes due to the absence of renormalization on the superconducting side of the interface. Another important difference from Eq. (7) is the presence of the off-diagonal term (10) in $\sigma_{D}$, which takes into account Friedel oscillations induced by Andreev reflection processes. Even though Andreev reflection can take place for energies above the gap, the combination $s$ is nonzero only for $D<\Delta$. Thus, the Andreev renormalization is effective only for subgap energies.

Equation (4), together with Eqs. (9) and (10), is the main result of the present work. The matrix $\sigma_{D}$ is obtained by differentiating the first order perturbation correction to the $S$ matrix

$$
\sigma_{D}=\frac{\partial}{\partial \ln D} \iint_{-\infty}^{0} \frac{d x d y}{i \hbar v_{F}} L_{k, x} \tilde{U}_{x, y}(D) L_{k, y},
$$

in the limit $\varepsilon_{k} \ll D$, where $\varepsilon_{k}=\hbar v_{F} k$. The transfer matrix $L_{k, x}$ describes free propagation of electron and hole quasiparticles

$$
L_{k, x}=\left(\begin{array}{cc}
e^{i\left(k_{F}+k\right) x} & 0 \\
0 & e^{-i\left(k_{F}-k\right) x}
\end{array}\right) .
$$

The first order molecular potential $\tilde{U}_{x, y}(D)$ in Eq. (11) depends on the cutoff and can be decomposed into Hartree and exchange terms $\tilde{U}_{x, y}=U_{x, y}^{H}-U_{x, y}^{\mathrm{ex}}$, that are given by

$$
\begin{aligned}
& U_{x, y}^{H}=\sigma_{z} \delta(x-y) \sum_{\sigma} \int_{-\infty}^{0} d z U_{x-z}\left\langle\Psi_{\sigma}^{\dagger}(z) \Psi_{\sigma}(z)\right\rangle, \\
& U_{x, y}^{\mathrm{ex}}=\frac{1}{2} U_{x-y}\left(\begin{array}{cc}
2\left\langle\Psi_{\uparrow}^{\dagger}(y) \Psi_{\uparrow}(x)\right\rangle & \left\langle\left[\Psi_{\uparrow}(x), \Psi_{\downarrow}(y)\right]\right\rangle \\
\left\langle\left[\Psi_{\downarrow}^{\dagger}(x), \Psi_{\uparrow}^{\dagger}(y)\right]\right\rangle & -2\left\langle\Psi_{\downarrow}^{\dagger}(x) \Psi_{\downarrow}(y)\right\rangle
\end{array}\right),
\end{aligned}
$$

where the square brackets denote the commutator. The operators $\Psi$ in Eq. (13) are taken in the scattering basis 


$$
\Psi_{\uparrow}(x)=\int \frac{d k}{\sqrt{2 \pi}}\left(e^{i\left(k_{F}+k\right) x} a_{\uparrow k}+e^{-i\left(k_{F}+k\right) x} b_{\uparrow k}\right),
$$

where the momentum integration is restricted to the interval ( $-D / \hbar v_{F}, D / \hbar v_{F}$ ), leading to the cutoff dependence of $\tilde{U}_{x, y}$ in Eq. (11). The operators $a_{\sigma k}$ and $b_{\sigma k}$ are related by the reflection matrix (8)

$$
\left(\begin{array}{c}
b_{\uparrow k} \\
b_{\downarrow-k}^{\dagger}
\end{array}\right)=r\left(\varepsilon_{k}\right)\left(\begin{array}{c}
a_{\uparrow k} \\
a_{\downarrow-k}^{\dagger}
\end{array}\right) .
$$

The angular brackets in Eq. (13) are defined by $\left\langle a_{\sigma k}^{\dagger} a_{\sigma^{\prime} k^{\prime}}\right\rangle=f\left(\varepsilon_{k}\right) \delta_{\sigma \sigma^{\prime}} \delta_{k k^{\prime}}$. Calculating $\sigma_{D}$ from Eq. (11) in the limit $\varepsilon_{k}, T \ll D$ we obtain the result (10).

Both the Hartree and exchange potentials in Eq. (13) are Hermitian, $\tilde{U}_{y, x}^{\dagger}=\tilde{U}_{x, y}$, and fulfill an electron-hole symmetry, $\sigma_{y} \tilde{U}_{x, y}^{*} \sigma_{y}=\tilde{U}_{x, y}$, for any $D$. Thus, both the unitarity as well as the electron-hole symmetry of the $S$ matrix are conserved under the RG flow (4).

The bare $S$ matrix for Andreev reflection has a specific energy dependence on the scale $\Delta$. For an ideal NS interface the bare Andreev reflection amplitude is given by

$$
\gamma_{\varepsilon}=\varepsilon / \Delta-\sqrt{(\varepsilon / \Delta)^{2}-1}, \quad \varepsilon>0,
$$

where the positive branch of the square root has to be chosen for $\varepsilon<\Delta$ and the relation $\gamma_{-\varepsilon}=-\gamma_{\varepsilon}^{*}$ extends the definition to negative energies. Furthermore, the reflection matrix (8) of a nonideal interface can be conveniently parameterized as [18]

$$
r^{N}=e^{i \theta_{1}} \frac{\left(1-\gamma_{\varepsilon}^{2}\right) \sqrt{R}}{1-\gamma_{\varepsilon}^{2} R}, \quad r^{A}=e^{i \theta_{2}} \frac{\gamma_{\varepsilon}(1-R)}{1-\gamma_{\varepsilon}^{2} R},
$$

where we assume the phases $\theta_{1,2}$ and the normal-state reflection coefficient $R \in(0,1)$ to be energy independent.

Because of the simple structure of $\Sigma_{D}$ in Eq. (9) it suffices to consider the reflection block of Eq. (4)

$$
\frac{\partial r_{D}(\varepsilon)}{\partial \ln D}=\sigma_{D}-r_{D}(\varepsilon) \sigma_{D}^{\dagger} r_{D}(\varepsilon) .
$$

In what follows we focus on the physically relevant limit of short-range interactions $d<\xi$ or, equivalently, $D_{0}>\Delta$, where $\xi=\hbar v_{F} / \Delta$ is the superconducting coherence length. We start from the analysis of Eq. (18) for the case of an ideal interface, $R=0$, and parameterize $r_{D}^{A}(\varepsilon)=$ $\exp \left(-i \phi_{D}(\varepsilon)\right)$, where the phase $\phi_{D}(\varepsilon)$ is real for $\varepsilon<\Delta$. The Andreev conductance below the gap is therefore not affected by interactions, while the Andreev phase $\phi_{D}(\varepsilon)$ strongly deviates from its elastic value $\arccos (\varepsilon / \Delta)$. Such an Andreev phase renormalization is absent in Refs. [13,14]. With the substitution

$$
\phi_{D}(\varepsilon)=\arccos \frac{\varepsilon / \Delta}{\cosh u_{D}-\sqrt{1-(\varepsilon / \Delta)^{2}} \sinh u_{D}}
$$

Eq. (18) is reduced to the differential equation for a function of one variable

$$
\frac{\partial u_{D}}{\partial \ln D}=\left(g_{1}(D)+g_{2}(D)\right) \tanh \left(u_{D}-w_{D}\right),
$$

with the initial condition $u_{\Delta}=0$, and with the notation $w_{D}=\operatorname{arctanh} \sqrt{1-(D / \Delta)^{2}}$. The solution to Eq. (20) in the limit $D \ll \Delta$ can be approximated by

$$
u_{D}=\left(\alpha_{2}-\alpha_{1} / 2\right) \ln \frac{\Delta}{D}+\frac{3}{4} \ln \left(1+a \ln \frac{\Delta}{D}\right)+\text { const, }
$$

where the parameter $a=2 \alpha_{1} /\left(1+2 \alpha_{1} \ln \left(D_{0} / \Delta\right)\right)$ has a logarithmic dependence on the initial cutoff $D_{0} \simeq \hbar v_{F} / d$. From Eq. (19) we obtain

$$
\cos \phi_{D}(\varepsilon) \simeq \frac{\varepsilon}{\Delta}\left(\frac{\Delta}{D}\right)^{\alpha_{2}-\alpha_{1} / 2}\left(1+a \ln \frac{\Delta}{D}\right)^{3 / 4},
$$

where $D=\max \left\{\varepsilon, k_{B} T\right\}<\Delta$. At zero temperature we let $D=\varepsilon$ in Eq. (22) and interpret the result as an additional suppression of the electron-hole coherence at finite $\varepsilon$. The effect of the Andreev phase renormalization can be seen most explicitly in the supercurrent or in measurements of the density of states. A detailed analysis of these quantities, though, is beyond the scope of the present work. We stress, however, that the renormalization (22) originates in the energy dependence of the bare $S$ matrix, which has been ignored in previous studies $[9,10]$.

For a nonideal interface, $R \neq 0$, Eq. (18) reduces to a set of four coupled RG equations for the variables $\gamma, R$, and $\theta_{1,2}$ in the parameterization (17). It is remarkable that for energies above the gap only one equation remains

$$
\frac{\partial R_{D}}{\partial \ln D}=\left(2 g_{1}-g_{2}\right) R_{D}\left(1-R_{D}\right) \frac{1-\gamma_{D}^{2}}{1-\gamma_{D}^{2} R_{D}},
$$

with the initial condition $R_{D_{0}}=R$ and with the function $\gamma_{D}$ given by the bare Andreev amplitude (16). Both the amplitude $\gamma_{\varepsilon}$ and the phases $\theta_{1,2}$ are not renormalized and do not acquire any $D$ dependence as far as $D>\Delta$. It, then, follows from Eq. (17) that $r^{N}(\Delta)=0$, hence the differential Andreev conductance at $e V=\Delta$ equals its elastic value $4 e^{2} / h$. On the other hand, renormalization (23) reduces to the result of Refs. [4,5] in the limit $\Delta \rightarrow 0$ and yields the well-known conductance suppression at low energies. Thus the interactions tend to sharpen the nonmonotonic behavior of the Andreev conductance near $e V=\Delta$ in accordance with Ref. [19].

For energies below the gap, Eq. (18) is equivalent to a joint renormalization of the three variables $\phi, R$ and $\theta_{1}$ in the parameterization (17), where $\phi$ is the Andreev phase, $\gamma=\exp (-i \phi)$. Thus, below the gap the renormalization cannot be reduced to that of the single parameter $R$ in contradiction to the phenomenological description of Ref. [20]. The phase $\theta_{2}$ is not renormalized in the entire energy range, since it can be removed by an appropriate gauge transformation. The Andreev conductance below the gap depends only on the absolute value of the amplitude $r_{D}^{A}(\varepsilon)$. One can prove from the explicit form of the RG 


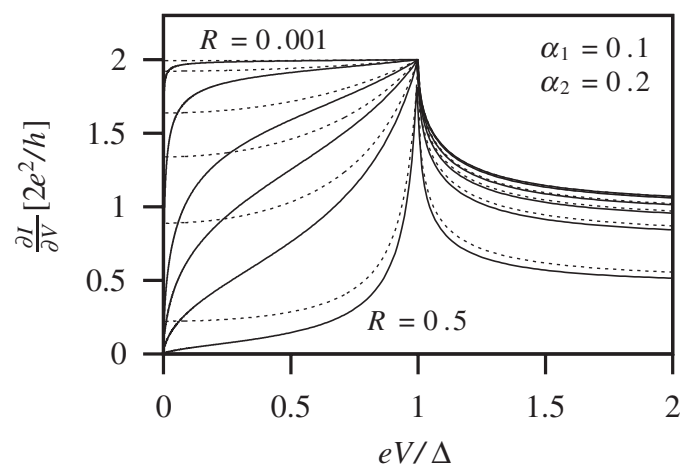

FIG. 1. Zero temperature differential Andreev conductance versus the voltage bias calculated from Eq. (1) with renormalized reflection amplitudes. The solid curves result from the numerical solution of Eq. (18) with $D_{0}=100 \Delta$ and correspond to different values of the normal-state resistance, $R=0.001,0.01,0.05,0.1$, $0.2,0.5$, and to the particular choice of constants $\alpha_{1}=0.1, \alpha_{2}=$ 0.2 . The dashed curves indicate the conductance in the absence of interactions.

equations that the energy dependence of $\left|r_{D}^{A}(\varepsilon)\right|$, unlike that of $r_{D}^{A}(\varepsilon)$, can be disregarded in the limit $\varepsilon \ll \Delta$. Therefore, for $D \ll \Delta$, we obtain from Eq. (18) $[13,14]$

$$
\frac{\partial\left|r_{D}^{A}\right|^{2}}{\partial \ln D}=2\left(2 \alpha_{2}-\alpha_{1}\right)\left|r_{D}^{A}\right|^{2}\left(1-\left|r_{D}^{A}\right|^{2}\right)
$$

which depends only on the RG-invariant combination $2 \alpha_{2}-\alpha_{1}$ of constants, which is positive for any repulsive interaction. The general solution of Eq. (24) can be written as

$$
\left|r_{D}^{A}\right|^{2}=\left[1+b(\Delta / D)^{2\left(2 \alpha_{2}-\alpha_{1}\right)}\right]^{-1},
$$

where the coefficient $b$ has to be determined from the solution of Eq. (18) for $\varepsilon, D \sim \Delta$. For a nearly ideal interface, $R \ll 1$, we find $b \propto R$, which allows us to estimate the width of the zero bias anomaly in the differential Andreev conductance as $\varepsilon_{c} \sim \Delta R^{1 /\left(4 \alpha_{2}-2 \alpha_{1}\right)}$.

We illustrate our findings in Fig. 1. The differential Andreev conductance at zero temperature is calculated from Eq. (1) with the renormalized amplitudes $r_{\varepsilon}^{N, A}(\varepsilon)$, which are found by the numerical solution of Eq. (18). The interaction-induced dip in the Andreev conductance has a width $e V_{c} \sim \varepsilon_{c}$, which, due to the power law dependence on $R$, is strongly sensitive to the normal-state resistance. Such sensitivity was indeed observed in the experiments by Morpurgo et al. [15] at low temperatures. At a large gate voltage - where the interface resistance was tuned to be low-a peak in the differential resistance was found, showing the suppression of the Andreev conductance due to the strong effect of electron-electron interactions. A small change in the gate voltage gave rise to a slight decrease of the normal-state resistance of the junction, which had a drastic effect and removed the interaction-induced suppression of the Andreev conductance.
In conclusion we have derived and analyzed the energydependent RG equations for the scattering matrix of an interacting normal-metal-superconductor interface. Our approach takes into account the intrinsic energy dependence of scattering at the NS interface and is readily generalized to SNS structures and to the case of a quasione-dimensional interacting normal metal. The effects of interaction on the Andreev conductance, but not on the Andreev phase, are shown to vanish in ideal NS junctions. Our results qualitatively explain recent experiments [15] with carbon nanotubes.

We acknowledge D. Aristov for drawing our attention to Ref. [16]. We also thank A. Furusaki, L. Glazman, and I. V. Gornyi for useful comments. This research was supported in part by the NSF under Grant No. PHY99-07949, the Swiss NSF, the DFG through No. SFB 513 and the BWResearch Network "Functional Nanostructures".

[1] A. F. Andreev, Zh. Eksp. Teor. Fiz. 46, 1823 (1964) [Sov. Phys. JETP 19, 1228 (1964)].

[2] G.E. Blonder, M. Tinkham, and T. M. Klapwijk, Phys. Rev. B 25, 4515 (1982).

[3] T. Giamarchi, Quantum Physics in One Dimension (Oxford University, New York, 2004).

[4] K. A. Matveev, D. Yue, and L. I. Glazman, Phys. Rev. Lett. 71, 3351 (1993).

[5] D. Yue, L. I. Glazman, and K. A. Matveev, Phys. Rev. B 49, 1966 (1994).

[6] C. L. Kane and M. P. A. Fisher, Phys. Rev. Lett. 68, 1220 (1992).

[7] A. Furusaki and N. Nagaosa, Phys. Rev. B 47, 4631 (1993).

[8] S. Vishveshwara, C. Bena, L. Balents, and M. P. A. Fisher, Phys. Rev. B 66, 165411 (2002).

[9] R. Fazio, F. W. J. Hekking, and A. A. Odintsov, Phys. Rev. Lett. 74, 1843 (1995); Phys. Rev. B 53, 6653 (1996).

[10] C. Winkelholz, R. Fazio, F. W. J. Hekking, and G. Schön, Phys. Rev. Lett. 77, 3200 (1996).

[11] Y. V. Nazarov and L. I. Glazman, Phys. Rev. Lett. 91, 126804 (2003).

[12] D. G. Polyakov and I. V. Gornyi, Phys. Rev. B 68, 035421 (2003).

[13] Y. Takane and Y. Koyama, J. Phys. Soc. Jpn. 66, 419 (1997).

[14] R. Fazio, F. W. J. Hekking, A. A. Odintsov, and R. Raimondi, Superlattices Microstruct. 25, 1163 (1999).

[15] A.F. Morpurgo, J. Kong, C. M. Marcus, and H. Dai, Science 286, 263 (1999).

[16] S. Lal, S. Rao, and D. Sen, Phys. Rev. B 66, 165327 (2002).

[17] J. Solyom, Adv. Phys. 28, 201 (1979).

[18] C. W. J. Beenakker, Rev. Mod. Phys. 69, 731 (1997).

[19] H.-W. Lee, H. C. Lee, H. Yi, and H.-Y. Choi, Phys. Rev. Lett. 90, 247001 (2003).

[20] H. T. Man, T. M. Klapwijk, and A. F. Morpurgo, cond-mat/ 0504566. 